\title{
Short-term power load forecasting based on BAT-BP neural network model
}

\author{
Jian Di \& Tao Yao \\ School of control and Computer Engineering, North China Electric Power of University, Baoding, Hebei \\ Province, 071000, China
}

\begin{abstract}
Accurate short-term forecasting of power load has important significance in Safe and stable operation and improvement of economic benefits of electric power system. The electric power system load forecasting usually adopts BP neural network (BPNN) method, but this method has slow convergence speed, is easy to fall in-to local minimum point and has poor robustness. In order to improve the accuracy of electric power load forecasting, the BA-BP load forecasting model of bat algorithm optimizing BPNN is proposed. Firstly, for each individual bat containing the BPNN parameters, the individual is encoded in a real number encoding, and the average relative error is set as the fitness function, and then get the Best bat individual by simulating the process of bats flying, So as to get the optimal parameter of BPNN. According to the optimal parameters to establish prediction model, finally, the performance test is carried out by simulation experiment, the contrast curve of training speed and relative error is obtained, the results proved that the BAT-BP prediction model has a significant advantage over the simple BP neural network, and it can improve the accuracy of the load forecasting results.
\end{abstract}

KEYWORD: Bat algorithm; BP neural network; Electric power load forecasting

\section{INTRODUCTION}

The short-term power load forecasting is mainly to predict the power load of a certain day or a few days in the future, it is a very important work for many electric power management departments in the whole power system. Power system load forecasting can be divided into two development stages, namely using the traditional method of forecasting and using modern intelligent methods to predict (Yang Tengdong, 2006), The traditional power load forecasting methods mainly include time series method, multivariate linear regression analysis method and pattern recognition method (CUI Wei, 2011),these traditional prediction method is simple and easy to implement, but cannot correctly describe the change rule of short-term load forecasting, however, Modern intelligent methods including neural networks and support vector machines, extreme learning machines and other machine learning methods are modeled based on nonlinear theory, which overcome the shortcomings of traditional power load forecasting methods, Because the power load is influenced by many factors, it has nonlinear characteristics, so it is very suitable to apply BP neural network to the load forecasting, which can improve the accuracy of power load forecasting (Zeng Ming, 2008).However, simple BP neural network in the training process need to repeatedly modify the network weights and threshold, often need to hundreds or even thousands of times of iteration may be completed before training, achieve the target error, which leads to large amount of calculation (Huang G B, 2006). Bat algorithm (Bat algorithm BA) is a meta heuristic optimization algorithm proposed by Yang in 2010, many domestic and foreign scholars have studied the algorithm, the results show that compared with other intelligent algorithms such as genetic algorithm, ant colony algorithm and neural network algorithm, new swarm intelligence algorithm--bat algorithm based on the simulated echo location in bats, will take into account local and global in solving a problem, and both can achieve mutual conversion in the dynamic search process, better to avoid falling into local optimal solution, has the better convergence (WANG $X$ S, 2012).So for BP neural network learning speed is slow and easy to fall into local minima and other characteristics, put forward a power load prediction model (BAT-BP), with bat algorithm to optimize the BP neural network, which can avoid original choice blindness of network initial weights and thresholds, in order to achieve the efficiency and accuracy of short term load forecasting of power system. 


\section{BAT ALGORITHM}

\subsection{Algorithm principle}

Bat algorithm: Bats use a sonar-echo locator, by detecting the time delay of emitted ultrasonic and echo, using the interaural time difference and the echo loudness change to build three-dimensional scenes of the surrounding environment (Yang X S, 2010), it is a random search algorithm to detect prey and avoid obstacles. The individual of the bat can be simulated by a series of behaviors such as the detection of prey, positioning and predation by ultrasonic wave, the behavior can be simulated by the following equation (Ye Z W, 2015).

When searching for prey, bats update the individual search frequency, speed and position according to the formula (1), formula (2) and formula (3):

$$
\begin{aligned}
f_{i} & =f_{\text {min }}+\left(f_{\max }-f_{\min }\right) \beta \\
v_{i}^{t} & =v_{i}^{t-1}+\left(x_{i}^{t-1}-x_{*}\right) f_{i} \\
x_{i}^{t} & =x_{i}^{t-1}+v_{i}^{t}
\end{aligned}
$$

$\beta$ is a random number in the range of $[0,1]$ and it is obey uniform distribution; $f_{i}$ is the search frequency of the bat $i$,it controls the range and speed of the bat individual movement, $f_{\min }$ and $f_{\max }$ are the minimum and maximum values of the search frequency respectively; $v_{i}^{t}$ and $v_{i}^{t-1}$ respectively represent the velocity of the bat $i$ at step $t$ and step $t-1 ; x_{i}^{t}$ and $x_{i}^{t-1}$ respectively indicate the position of the bat $\mathrm{i}$ in step $\mathrm{t}$ and step $\mathrm{t}-1 ;{ }^{X_{*}}$ represents the current global optimal position at step $\mathrm{t}$.

Generating random number rand, obey uniform distribution, If rand $>$ Pulse frequency $r_{i}$, Perturbing the current optimal solution randomly, generating a new local solution and processing the new crossborder solution.

$$
x_{\text {new }}=x_{\text {old }}+\varepsilon A^{t}
$$

$\varepsilon$ is a random number in the range of[-1,1],and it can control the intensity and direction of random walk; $A^{t}$ represents the average loudness of all bats in the same period at present.

In the process of searching for prey, bats emit ultrasonic pulses will large loudness and low frequency at the initial stage, it is conducive to search in the broader space, after the bat discovers the prey, it will reduce the loudness pulse gradually as well as increases the pulse frequency in order to accurately grasp the position of the prey in the search space. Pulse loudness and pulse frequency are updated by the formula (5) and (6).

$$
\begin{aligned}
& A_{i}^{t+1}=\alpha A_{i}^{t} \\
& r_{i}^{t+1}=r_{i}^{0}[1-\exp (-\gamma t)]
\end{aligned}
$$

Among them, $A_{i}^{t+1}$ and $A_{i}^{t}$ respectively indicate the pulse loudness of the bat $\mathrm{i}$ in step $\mathrm{t}+1$ and step t; $\alpha \in[-1,1]$,is pulse loudness attenuation coefficient; $r_{i}^{t+1}$ indicates pulse frequency of the bat $\mathrm{i}$ in step $\mathrm{t}+1 ; r_{i}^{0}$ indicates maximum pulse frequency of the bat $\mathrm{i} ; \gamma>0$,is pulse frequency enhancement coefficient. It is easy to see, $t \rightarrow \infty, A_{i}^{t} \rightarrow \infty, r_{i} \rightarrow r_{i}^{0}$. The flow chart of the bat algorithm is shown in Figure 1.

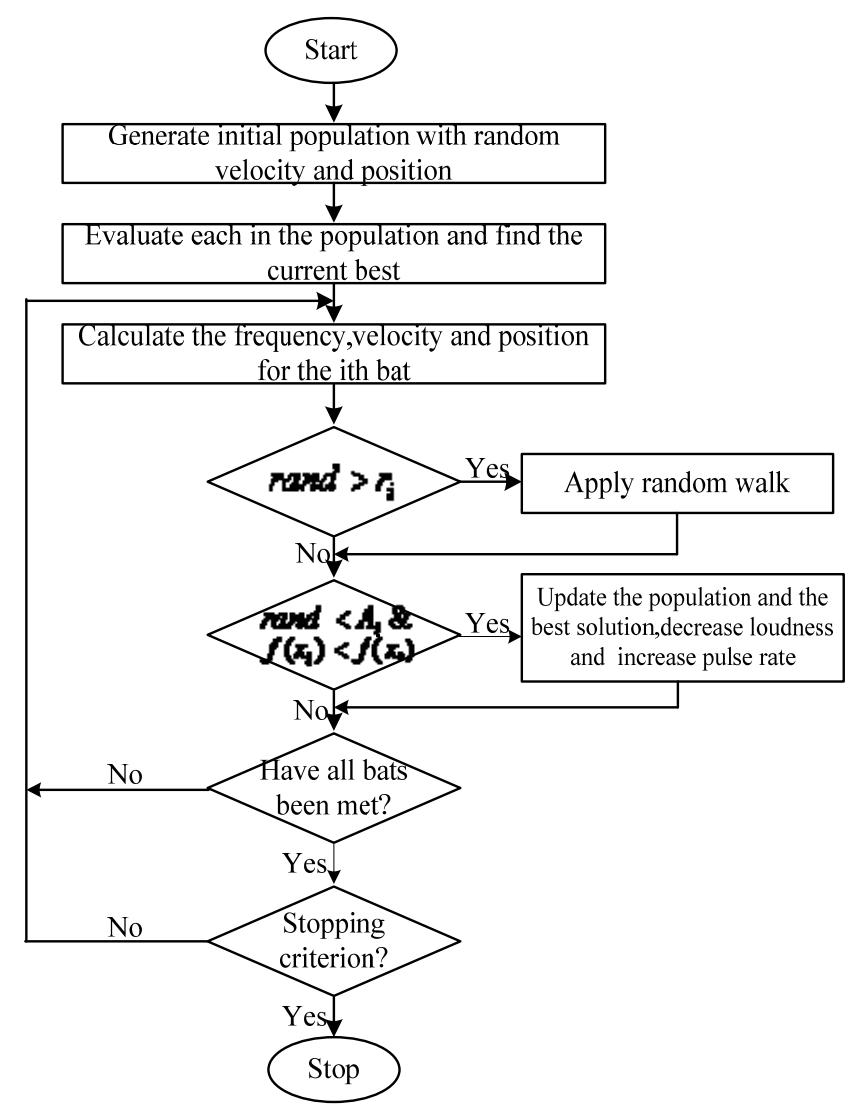

Figure 1. Bat algorithm flow chart

\subsection{Algorithm performance}

In order to evaluate the Optimization performance of the proposed bat algorithm, two standard test functions are selected to carry out the simulation experiment, and compared with the basic particle swarm algorithm and genetic algorithm. Two test functions are shown in the formula (7) and (8), The detailed description of each test function are shown in the table1:

$$
\begin{aligned}
& f_{1}(x)=\frac{\sin ^{2} \sqrt{x_{1}^{2}+x_{2}^{2}}-0.5}{\left[1+0.001\left(x_{1}^{2}+x_{2}^{2}\right)\right]^{2}}-0.5 \\
& f_{2}(x)=\sum_{i=1}^{5} i \times \cos \left((i+1) \times x_{1}+i\right) \times \\
& \sum_{j=1}^{5} j \times \cos \left((j+1) \times x_{2}+j\right)
\end{aligned}
$$


Table 1. Test function description

\begin{tabular}{lccl}
\hline $\begin{array}{l}\text { Function } \\
\text { name }\end{array}$ & function & $\begin{array}{l}\text { search } \\
\text { space }\end{array}$ & $\begin{array}{l}\text { optimal } \\
\text { solution }\end{array}$ \\
\hline $\begin{array}{l}\text { Schaffer } \\
\text { F6 }\end{array}$ & $f_{1}(x)$ & {$[-100,100]$} & $f_{1}(0,0)=-1$ \\
\hline Shubert & $f_{2}(x)$ & {$[-10,10]$} & $\begin{array}{l}f_{2}\left(x^{*}\right) \\
=-1867309\end{array}$ \\
\hline
\end{tabular}

BA refers to the bat algorithm, in the bat algorithm, the search frequency $f$ drop in the range of $[0,2]$,the maximum pulse frequency $r^{0}=0.75$, the maximum pulse loudness $A=0.25$, the loudness pulse attenuation coefficient $\alpha=0.94$, the pulse frequency increase coefficient $\gamma=0.05$.PSO refers to the basic particle swarm algorithm, in the particle swarm optimization algorithm, the inertia weight factor in a linear reduction is proposed in the reference (Shi Y, 1998), $w_{\max }=0.9, w_{\min }=0.4$, learning factors $c_{1}=c_{2}=1.4962$. GA refers to the standard genetic algorithm, In the genetic algorithm, the chromosome adopts the binary encoding method and the elite reservation method, the retention ratio of the elite individual is $5 \%$, the crossover probability $p_{c}$, the mutation probability $p_{m}$ are respectively 0.40 and 0.07 .The maximum number of iterations of the above three algorithms ITER_Max $=300$, The dimension of search space $d=2$, the population number $n=20$, The number of independent operation of each algorithm is 20 times. The results of the experiment are shown in table 2.

Table 2. Search results of each algorithm

\begin{tabular}{|c|c|c|c|}
\hline \multirow{2}{*}{ function } & \multicolumn{3}{|c|}{ Average optimal value / (optimal rate) } \\
\cline { 2 - 4 }$f_{1}(x)$ & GA & PSO & BA \\
\hline $\begin{array}{c}0.9805 \\
(0 \%)\end{array}$ & $\begin{array}{c}0.9662 \\
(42.3 \%)\end{array}$ & $\begin{array}{c}0.9927 \\
(83.2 \%)\end{array}$ \\
\hline$f_{2}(x)$ & $\begin{array}{c}182.0910 \\
(0 \%)\end{array}$ & $\begin{array}{c}167.2571 \\
(47.9 \%)\end{array}$ & $\begin{array}{c}181.1619 \\
(93.5 \%)\end{array}$ \\
\hline
\end{tabular}

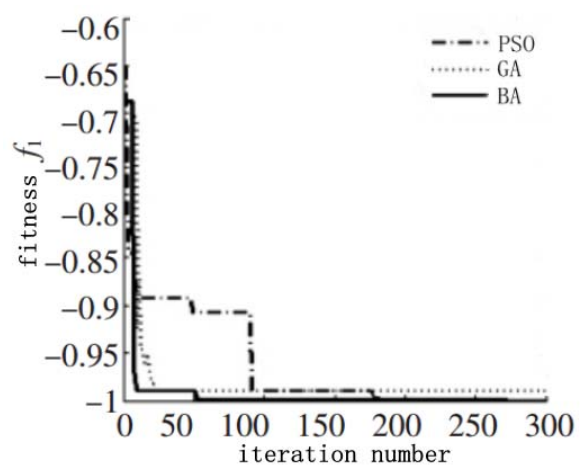

Figure 2. Schaffer F6 function optimization curve

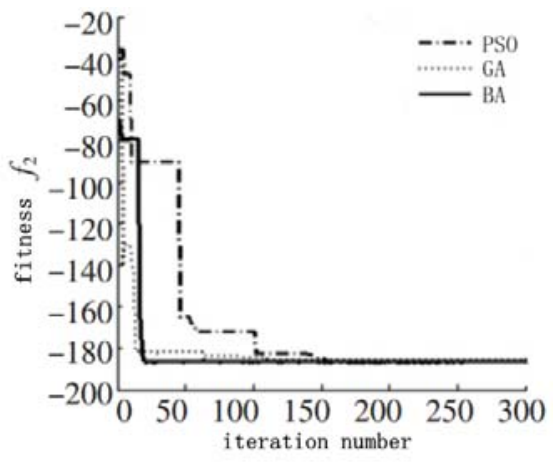

Figure 3. Shubert function optimization curve

Test results show that, for function $f_{1}$ and $f_{2}$, PSO algorithm and BA are both convergent to the optimal solution, But the accuracy and optimization rate of BA are higher than that of PSO algorithm. The fitness function contrast curve is shown in figure 2 and figure3.

\section{BAT-BP POWER LOAD FORECASTING MODEL}

Although the nonlinear learning ability of BPNN multilayer perceptron is strong and it is widely used in the field of power load forecasting, there are still many defects, For example, (1)the initial weight and the threshold vector are random, which Causes the training speed is not sure and the training result does not converge;(2)The gradient descent method is easy to make the BPNN into the local best, so that the fitting effect is good and the forecast ability is poor. In view of the defects of BPNN, a new swarm intelligence algorithm--bat algorithm is proposed to optimize the parameters of the BPNN algorithm. The BAT-BP power load forecasting model is established to improve the convergence speed and prediction accuracy.

\subsection{Bat algorithm optimized BP neural network}

The specific process of optimizing the BP neural network with the bat algorithm:

Coding. Each bat individual contains all the weights and the threshold of the BP neural network, encoded by the real number coding method.

Population initialization. Randomly generated N bats in d-dimensional search space and form the initial bat populations, including the random initialization of bat position $x_{i}$, velocity $v_{i}$, the Search pulse frequency range $\left[f_{\min }, f_{\max }\right]$,pulse frequency enhancement coefficientt $\gamma$, pulse loudness $A_{i}$, maximum loudness $A^{0}$ and attenuation coefficient of loudness $\alpha$, pulse frequency $r_{i}$, maximum pulse frequency $r^{0}$, maximum iterative number iter_max. Set $\mathrm{n}=10, \mathrm{~d}=5$ here. 
The fitness function $f(x)$ can be set as the average relative error function. The smaller the fitness value is, the better the location of bat individual is. The greater the fitness value is, the worse the individual position is. According to the size of the fitness value , looking for the current global optimal location.

According to the formula (1) (3) to update the search frequency, velocity and position of the bat individual. Evaluate each bat individual, and get the best position in the whole.

Generate a random number Rand, if the rand $>r_{i}$ ( $r_{i}$ is the ith bats pulse frequency), Get a new solution $x_{\text {new }}$ by formula (4).

Generate a random number rand, if rand $<A_{\text {i }}$ and $f\left(x_{i}\right)<f\left(x_{*}\right)$, then update bat individual, and reduce the pulse loudness by formula(5),increase pulse frequency by formula(6).

Calculate the fitness value of all bats, the lowest fitness value is the global optimal position. If the minimum fitness value achieve the network training accuracy or achieving the maximum number of iterations, then ended the iteration and turn to steps 8); otherwise turn to step 4) to continue to update the velocity and location of the bat.

Outputing the weight and threshold value of the optimal bats which are the initial weight and threshold of the BP neural network. By using the BP neural network optimized by bat algorithm establish the optimal BAT-BP power load forecasting model.

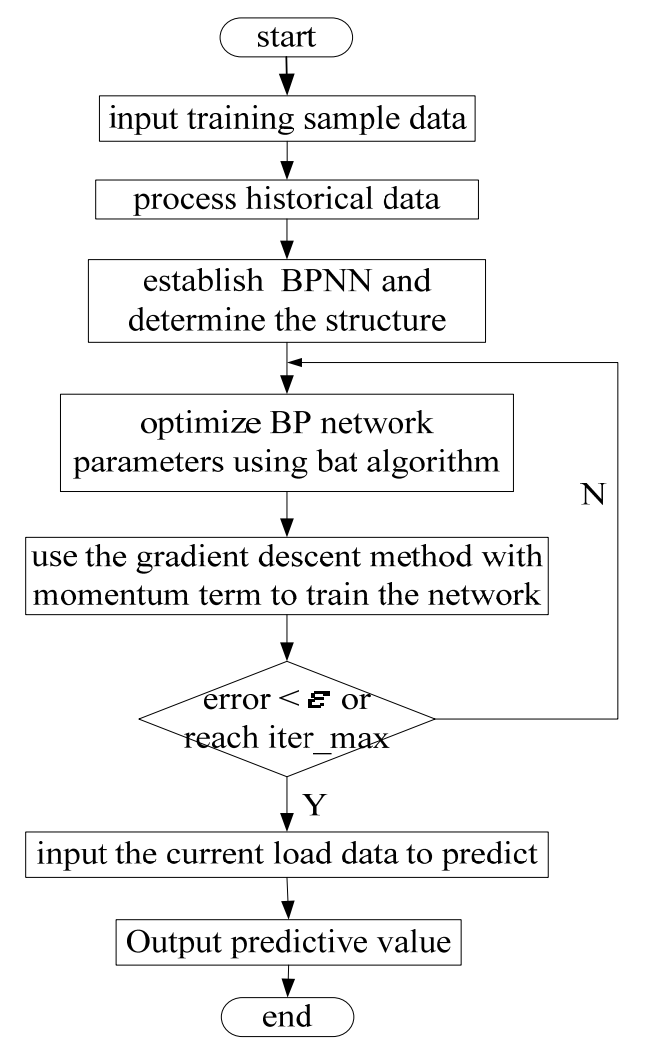

Figure 4. BAT-BP load forecasting process

\subsection{BAT-BP model prediction process}

The power load data provided by the EUNITE competition is used as the experimental sample, including historical power load data--the daily 24 hour power load data From March 30, 1998 to April 23, 1998; daily average temperature data; date type data. The forecasting process of BAT-BP power load forecasting model is shown in figure4:

\section{SIMULATION EXPERIMENT}

\subsection{Experimental environment}

The hardware environment is notebook computer with Intel Core Duo CPU, 2.20GHz frequency, 4G memory and 500G hard disk, software environment is Windows 7 operating system and software is MATLAB 2014R,This paper using Matlab bp neural network toolbox function to programme and to complete BP neural network load forecasting model construction, training and prediction simulation.

\subsection{Evaluation criteria of prediction model}

To verify the effectiveness of proposed BAT-BP power load forecasting modal, Compared with the result of the classical BP neural network (BPNN),BP neural network optimized by genetic algorithm (GABPNN), BP neural network optimized by particle swarm algorithm(PSO-BPNN). Evaluation criteria for model performance are as shown in formula (9) and (10):

1) relative error

$$
R E=\frac{\left|y_{i}-y_{i}^{\prime}\right|}{y_{i}} \times 100 \%
$$

2) Average relative error

$$
\text { ARE }=\frac{1}{n} \sum_{i=1}^{n} \frac{\left|y_{i}-y_{i}^{\prime}\right|}{y_{i}} \times 100 \%
$$

Here $y_{i}$ and $y_{i}^{\prime}$ respectively represent the actual load value and the predicted load value.

\subsection{Simulation results analysis}

1) The convergence results of classical BP neural network are shown in Figure 5. The figure shows the variation of the training error with the increase of the number of iterations, but when reached the maximum number of iterations 10000 times, the training error of BPNN is 0.00268103 , did not reach the target requirements of 0.001 .

When the bat algorithm is used to optimize the BPNN, the convergence results are shown in Figure 6 . The figure shows that when the number of iterations is 4635, the training error curve converges to 0.000999956, and the target error is 0.001. By comparing the variation of the two training error curves, 
the bat algorithm optimized BP neural network greatly improves the convergence speed.

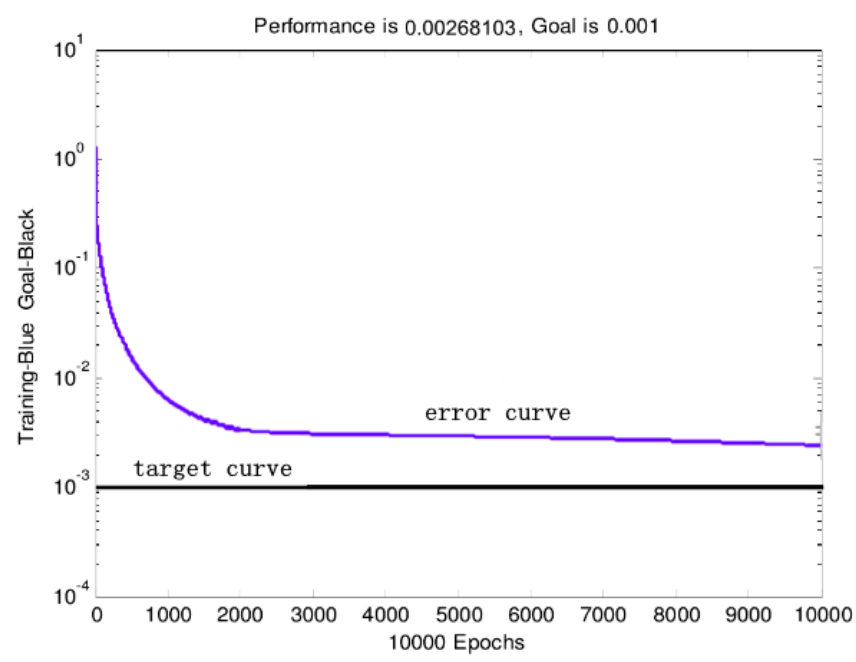

Figure 5. BPNN without optimization Convergent result

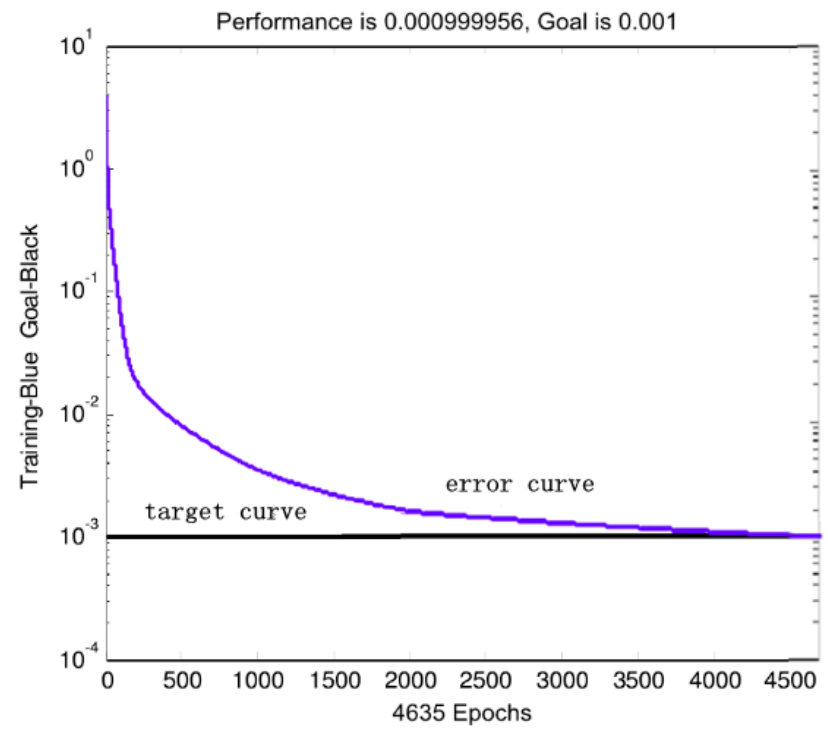

Figure 6. BPNN optimized by bat algorithm convergence results

2)To demonstrate the performance of BAT-BP load forecasting model established, we compared its load forcasting results with the GA-BPNN and PSOBPNN models. Figure 7 and figure 8 are the prediction results and relative error of above load forecasting models. Error analysis of each model in 24 times are shown in table 3.

Analysis the above figures and tables, prediction results of the BAT-BP load forecasting model is more close to the actual load. The maximum relative error of forecasting model of the BPNN without optimization and the GA-BPNN were $4.1 \%$ and $3.21 \%$ respectively, the minimum relative error of the classical BPNN is 3.05\%, which exceeds the prescribed prediction accuracy. However, the minimum relative error、 the maximum relative error and the average relative error of BAT-BP were $1.26 \%$, 1.87\%, $1.55 \%$, respectively, which were less than PSO-BP minimal, maximal and relative error, indicating that BAT-BP load forecasting model is not only meets the prediction accuracy of $3 \%$, but has a higher prediction accuracy.

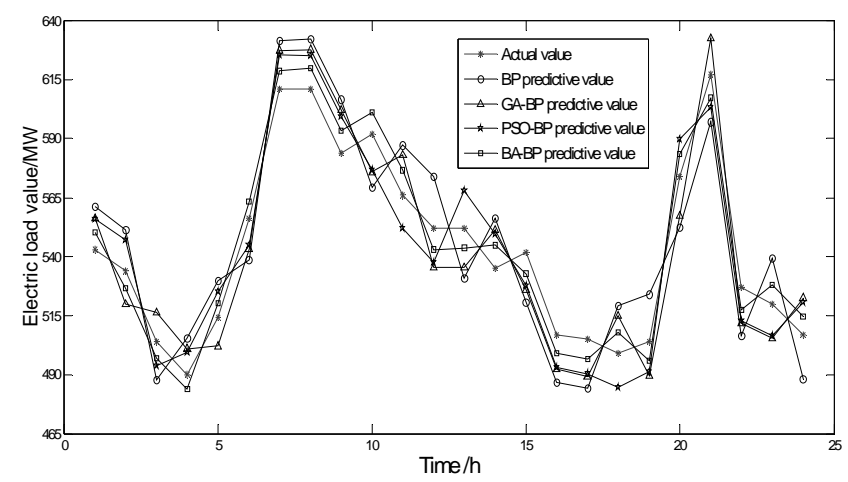

Figure 7. prediction results of each modal

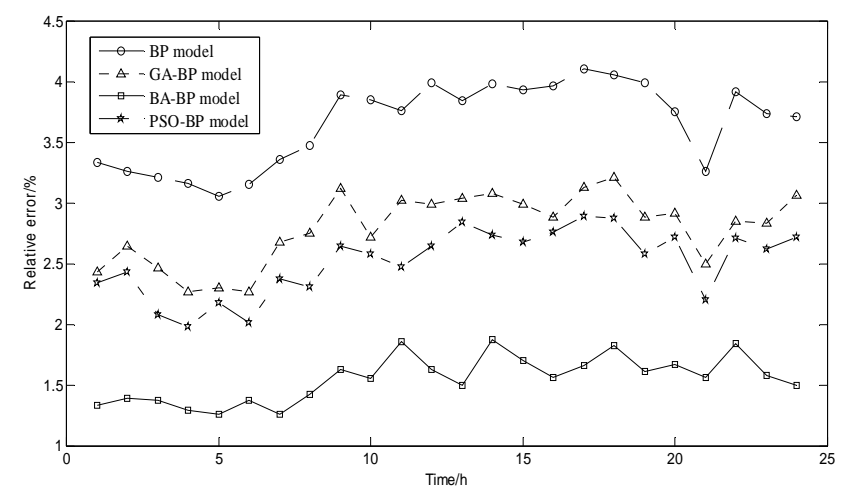

Figure 8. relative error of each modal

Table 3. Relative error analysis of each model

\begin{tabular}{llll}
\hline prediction model & RE_MIN(\%) & RE_MAX(\%) & ARE(\%) \\
\hline BPNN & 3.05 & 4.10 & 3.65 \\
GA-BPNN & 2.27 & 3.21 & 2.79 \\
PSO-BPNN & 1.98 & 2.89 & 2.52 \\
BA-BPNN & 1.26 & 1.87 & 1.55 \\
\hline
\end{tabular}

\section{CONCLUSION}

In this paper, BP neural network initialization parameters are random, leading to slow convergence and local minimum points. Therefore, the optimization of BP neural network by bat algorithm is used to get the optimal weights and thresholds, and the optimized BPNN is used for short-term power load prediction. Simulation experimental results show that with the optimization, the overall performance of the BP neural network increased, and comparing with the GA-BP and PSO-BP neural network load forecasting model, BAT-BP has more excellent power load forecasting results. In short, The BP neu- 
ral network model which is optimized by bat algorithm has faster convergence speed and higher prediction accuracy, can predict short-term power load very well, has a widely used in the power system required high accuracy.

\section{REFERENCE}

CUI Wei,ZHANG Mei.Prediction of load based on grey theory and BP networks[J].Journal of Liaoning Technical University(Natural Science),2011,30(4):631-633.

Huang G B, Zhu Q Y, Siew C K. Extreme learning machine: theory and applications[J]. Neurocomputing, 2006, 70(1): 489-501.

Huang Xin,Zhao Jincheng,Xie PU.Simulation of power station load forecasting based on BP neural network[J].Foreign electronic measurement technology,2012,31(11): 41-43.

Shi Y, Eberhart R. A modified particle swarm optimizer[C]//Evolutionary Computation Proceedings,1998.

IEEE World Congress on Computational Intelligence., The 1998 IEEE International Conference on. IEEE, 1998: 69-73.

Song Lili, Shi Li.Model analysis of college students teacher ratio prediction based on the BP network optimized by genetic algorithm[J].Journal of ShenYang Ligong University,2013 (1):80-84.

WANG X S,GANDOMI A H.Bat algorithm: a novel approach for global engineering optimization[J].Engineering Computation,2012,29(5):267-289.

Yang Tengdong,Peng Zhiwei.Analysis and application of common methods of power load forecasting[J].Guangxi Electric Power,2006(9):83-86.

Yu-ming G A O, Ren-jin Z.House price forecasting based on genetic algorithm and BP neural network [J].Computer engineering,2014,40(4):187-191.

Yang X S. A new metaheuristic bat-inspired algorithm[M]// Nature inspired cooperative strategies for optimization (NICSO 2010). Springer Berlin Heidelberg, 2010: 65-74.

Ye Z W, Wang M W, Liu W, et al. Fuzzy entropy based optimal thresholding using bat algorithm[J]. Applied Soft Computing, 2015, 31: 381-395.

Zeng Ming, Liu Baohua, Xu Zhiyong,et al.Short term load forecasting based on chaotic fuzzy neural network method [J].Journal of Hunan University: Natural Science edition,2008,35(1):58-61. 